\title{
Experimental and numerical investigation of the effective electrical conductivity of nitrogen-doped graphene nanofluids
}

\author{
Mohammad Mehrali · Emad Sadeghinezhad · Mohammad Mehdi Rashidi · \\ Amir Reza Akhiani - Sara Tahan Latibari · Mehdi Mehrali · \\ Hendrik Simon Cornelis Metselaar
}

Received: 17 January 2015/Accepted: 2 June 2015/Published online: 13 June 2015

(C) Springer Science+Business Media Dordrecht 2015

\begin{abstract}
Electrical conductivity is an important property for technological applications of nanofluids that have not been widely investigated, and few studies have been concerned about the electrical conductivity. In this study, nitrogen-doped graphene (NDG) nanofluids were prepared using the two-step method in an aqueous solution of $0.025 \mathrm{wt} \%$ Triton $\mathrm{X}-100$ as a surfactant at several concentrations $(0.01$, $0.02,0.04,0.06 \mathrm{wt} \%)$. The electrical conductivity of the aqueous NDG nanofluids showed a linear dependence on the concentration and increased up to $1814.96 \%$ for a loading of $0.06 \mathrm{wt} \%$ NDG nanosheet. From the experimental data, empirical models were
\end{abstract}

M. Mehrali (更) A A. R. Akhiani - S. Tahan Latibari · M. Mehrali - H. S. C. Metselaar

Department of Mechanical Engineering and Advanced Material Research Centre, University of Malaya,

50603 Kuala Lumpur, Malaysia

e-mail: mohamad.mehrali@siswa.um.edu.my

E. Sadeghinezhad ( $\square)$

Department of Mechanical Engineering, University of Malaya, 50603 Kuala Lumpur, Malaysia

e-mail: esn802001@yahoo.com

M. M. Rashidi

Shanghai Automotive Wind Tunnel Center, Tongji University, 4800 Caoan Rd., Jiading, Shanghai 201804, China

M. M. Rashidi

ENN-Tongji Clean Energy Institute of Advanced Studies, Shanghai, China developed to express the electrical conductivity as functions of temperature and concentration. It was observed that increasing the temperature has much greater effect on electrical conductivity enhancement than increasing the NDG nanosheet loading. Additionally, by considering the electrophoresis of the NDG nanosheets, a straightforward electrical conductivity model is established to modulate and understand the experimental results.

Keywords Nitrogen-doped graphene $\cdot$ Nanofluid . Characterization · Stability · Electrical conductivity · Empirical models

\section{Introduction}

Energy transport is an integral part of a wide range of research areas, including chemical industry, oil and gas, nuclear energy, electrical energy, etc. In previous decades, ethylene glycol (EG), oil, and water were used as heat transfer fluids (Mehrali et al. 2014b; Sadeghinezhad et al. 2014). However, development of heat transfer fluids with improved thermal conductivity has become more and more critical to the performance of energy systems (Safaei et al. 2014). The convective heat transfer coefficients and pumping power requirements of fluids depend strongly on the Prandtl number and are highly influenced by viscosity and temperature of the fluid (Sadeghinezhad et al. 
2014; Togun et al. 2014). On the other hand, dispersion of nanoparticles is a challenging task in the preparation of nanofluids and the nature of the surfactant plays a crucial role in the reaction mechanism. The first step towards preparation of a stable suspension was to find a suitable surfactant. Gum arabic (GA), sodium dodecyl sulfate (SDS), hexadecyltrimethylammonium bromide (CTAB), and Triton $\mathrm{X}-100$ were used to produce aqueous nanofluids suspensions (Banerjee and Krupanidhi 2010). In addition, it can be noted that the importance of electrical conductivity characteristics of nanoparticle suspensions has largely been ignored in most studies and a few research works have been done on the electrical properties of nanofluids. It can be noted that most of the studies have typically investigated the mechanism of heat stability, thermal properties, and heat transfer characteristic of the nanofluids and their superior thermal performance over the conventional heat transfer systems.

Yet, apart from the thermophysical properties, the electrical conductivity might bring useful information on the state of dispersion and performance of the nanofluids (Ganguly et al. 2009). Electrical conductivity $(\sigma)$ is the ability to conduct an electric current, which in a liquid solution is a liquid solution carried by anions and cations. Electrical conductivity is one of the important material characteristics for application in various fields and it is different and unique for each material and depends on the background electrolyte and particle size, charge, and concentrations. Recently, development of nanomaterials research has provided great changes in sciences and technology. Additionally, the electrical conductivity of nanofluids still remains poorly understood compared to the thermophysical properties of nanofluids (Chakraborty and Padhy 2008). The electrical conductivity of a suspension can either increase or decrease depending on the size, concentration, and background of electrolyte of nanoparticles. Electrical conductivity is normally measured in aqueous solutions of electrolytes, which can be either strong or weak and dependent on the temperature (White et al. 2011) Based on the surface and colloid theory, there is an electrical double layer (EDL) around each particle, which is a major factor in the colloidal stability of suspensions. The effect of EDL can be understood by the zeta potential, which is studied in the $\mathrm{pH}$ selection of the flotation process (Dong et al. 2013). The EDL theory is useful in the explanation of the electrostatic and electrophoretic properties of suspensions. Some researches show that the electrical conductivity can affect the heat transfer properties of the nanofluids (electrical phenomena at interfaces: fundamentals: measurements, and applications 1998; Kalteh et al. 2011). The study of this property is important for applications which require electrically conducting fluids, including field-induced pattern formation in colloidal dispersions and electrically conducting adhesive technology.

The dispersed nanoparticles including $\mathrm{ZnO}, \mathrm{Cu}$, $\mathrm{CuO}, \mathrm{TiO}_{2}, \mathrm{Al}_{2} \mathrm{O}_{3}$, and CNT gain surface charge due to the protonation or deprotonation of a surface group such as a hydroxyl ligand (-OH) (White et al. 2011). The surface charge of nanoparticles can be adjusted by chemical treating of the nanoparticles surface or electrolyte solutions by altering the $\mathrm{pH}$ of the suspension. Several researchers have studied on the electrical conductivity measurements and found large enhancement in the electrical conductivity of nanofluids compared to the base fluid as the temperature concentration are increased (Cruz et al. 2005; Fang and Zhang 2005; Ganguly et al. 2009; Lisunova et al. 2006; Wong and Bhshkar 2006). Ganguly et al. (2009) found a factor of 150 for enhancement in electrical conductivity for $\mathrm{Al}_{2} \mathrm{O}_{3}$ nanofluids at a volume fraction of $3 \%$. Additionally, they found that the enhancement in electrical conductivity has a factor of 100 greater than the predicted value by the Maxwell model (Maxwell 1881; Maxwell and Thompson 1904). On the other hand, there are no available models for the electrical conductivity of nanofluids, and the researchers have simply used a linear curve fit without physical interpretation (Goharshadi et al. 2014; White et al. 2011). Kole and Dey (2013) have studied on the electrical conductivity of graphene nanofluid (70:30 mixture of DW and EG) and found that the electrical conductivity increased linearly with temperature and concentrations. Goharshadi and Azizi-Toupkanloo (2013) have measured the electrical conductivity of silver nanofluid and they observed $39.75 \%$ increase in the electrical for a volume fraction of $2 \%$ at $50{ }^{\circ} \mathrm{C}$. Azizi-Toupkanloo et al. (2013) have measured the electrical conductivity of nanofluids of $\mathrm{Pd} / \mathrm{Ag} \mathrm{NPs}$ at different mass fractions and temperatures. They found that electrical conductivity of DW at $25{ }^{\circ} \mathrm{C}$ was increased $38.41 \%$ when $1 \% \mathrm{Pd} / \mathrm{Ag}$ NPs was added. 
Recently, graphene is one of the amazing recent developments in modern science and one of the most promising materials for implementation in the next generation electronic devices (Usachov et al. 2011). Doping is a common approach to tailor the electronic properties of the semiconductor materials. For instance, after doping with $\mathrm{N}$ or $\mathrm{B}$ atoms, these semiconductor materials become n-type or p-type, respectively. Doping can also dramatically alter the electrical properties of graphene (Wei et al. 2009). Due to these reasons and based on the literature, NDG nanosheet has high electrical conductivity. On the other hand, little research have been done on the electrical conductivity of NDG nanofluids.

The objective of this work was to experimentally investigate the influence of nanosheet concentration and temperature on the electrical conductivity of aqueous NDG nanofluids. Recent reports have shown the effect of NDG roles in many technologies, industrial applications, and electrochemical devices such as batteries, super capacitors, etc. (Reddy et al. 2010). In the present work, the NDG synthesized by heat treatment of graphene in ammonia solution was followed by the preparation of stable nanofluids with desired characteristics. The present report contains results on the stability and electrical conductivity at different concentrations of the NDG nanofluids. Results are discussed to identify the mechanisms

responsible for the electrical conductivity of NDG nanofluids prepared with different amounts of NDG nanosheets in distilled water $(0.01,0.02,0.04$, and $0.06 \mathrm{wt} \%$ ) with Triton $\mathrm{X}-100$ as a surfactant.

\section{Materials and methods}

\section{Synthesize of nitrogen-doped graphene}

A simplified Hummers' method was used to synthesize graphene oxide (GO) (Mehrali et al. 2013a, 2014a) and the NDG was prepared by a hydrothermal process with GO as raw material in an ammonia solution. As shown in Fig. 1, a mixture of $50 \mathrm{mg}$ of $\mathrm{GO}$ and $100 \mathrm{~mL}$ of $\mathrm{H}_{2} \mathrm{O}$ was sonicated for $1 \mathrm{~h}$ and $\mathrm{pH}$ of the solution was adjusted to 11 using ammonia. This homogenous solution was hydrothermally treated in a Teflon-lined autoclave at a temperature of $160{ }^{\circ} \mathrm{C}$ for $12 \mathrm{~h}$. A black wooly precipitate was collected with centrifugation, followed by washing with deionized water. Finally, the obtained NDG samples were dried at $50{ }^{\circ} \mathrm{C}$ under vacuum.

Nanofluid preparation

Water is a common heat transfer fluid. However, NDG cannot be directly dispersed in water, as there is no chemical affinity between them. Mixing of these materials directly leads to non-uniform suspensions and sedimentation of NDG starts almost immediately. Dispersion of NDG is a challenging task in the preparation of nanofluids. The nanofluid should be a stable and an agglomerate-free suspension without sedimentation for a long duration. In this experiment, the most effective surfactant and sonication time were selected by examining the stability of nanofluids. The first step towards preparation of a stable suspension was to find a suitable surfactant. According to previous research (Yousefi et al. 2012), surfactant and sonication time are important parameters for dispersing the nanosheet. Based on Yousefi et al.'s (2012) study, Triton $\mathrm{X}-100$ as a non-ionic surfactant is the best dispersing agent for carbon-based nanoparticles due to the presence of benzene ring. This benzene ring is adsorbed on the NDG surface due to $\pi-\pi$ stacking. In this, Triton X-100 could help nanosheet dispersion by forming a large solvation shell around them. The optimized parameters for the NDG nanofluid preparation were $60 \mathrm{~min}$ ultrasonication (probe) and $0.025 \mathrm{wt} \%$ aqueous solution of Triton $\mathrm{X}-100$ as a surfactant, and then the concentrations of nanofluids were maintained at $0.01,0.02,0.04$, and $0.06 \mathrm{wt} \%$.

Measurements procedure of the electrical conductivity

In this experiment, measurements of the electrical conductivity were carried out both as a function of nanosheet concentration and temperature. In this experiment, the electrical conductivity was measured using a bench type of electrical conductivity meters. The electrical conductivity measurements of NDG nanofluid were conducted to examine the effects of variations in the temperature range from 25 to $60{ }^{\circ} \mathrm{C}$ and concentration between 0.01 and $0.06 \mathrm{wt} \%$. For each case, six measurements were performed, and the mean value was reported. 


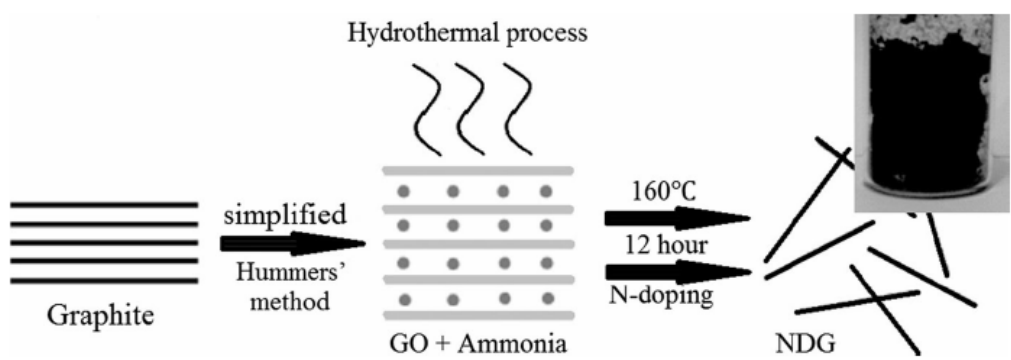

Fig. 1 Schematic illustration of nitrogen-doped graphene synthesize

Analysis methods

Field emission scanning electron microscopy (FESEM-CARL ZEISS-AURIGA 60) was used to observe the microstructure of the NDG. Transmission electron microscopy (TEM) measurements were conducted on a CARL ZEISS-LIBRA120 microscope. An $\mathrm{X}$-ray photoemission spectrometer (PHI-Quantera II) with an $\mathrm{Al}-K \alpha(h v=1486.8 \mathrm{eV}) \mathrm{X}$-ray source was used to identify bonding of the elements in the NDG. $\mathrm{X}$-ray diffraction (XRD) patterns were measured on the Empyrean PANALYTICAL diffractometer. Raman spectra were obtained using a Renishaw Invia Raman Microscope using laser excitation at $514 \mathrm{~nm}$. Atomic force microscopy (AFM, Bruker- MultiMode 8) in tapping mode was used to show the size of GO and NDG. Fourier transform-infrared (FT-IR) absorption spectra of the composites were recorded using a Bruker FT-IR (Bruker Tensor 27) spectrometer at room temperature in the range $4000-400 \mathrm{~cm}^{-1}$ using ATR mode. The Brunauer-Emmett-Teller method (BET-Autosorb-iQ2) was used to measure specific surface area and pore distribution of the NDG sample. The weight loss and thermal stability of PCMs are obtained by thermogravimetric analysis (METTLER TOLEDO SDTA 851-Error $\pm 5 \mu \mathrm{g}$ ) at a heating rate of $10{ }^{\circ} \mathrm{C} / \mathrm{min}$ and a temperature of $50-500{ }^{\circ} \mathrm{C}$ in purified nitrogen atmosphere. The zeta potential of the nanofluids was measured on a Zetasizer nano (Malvern Instruments Ltd., United Kingdom). The rheological behavior of nanofluids with amounts of NDG was measured on Anton Paar rheometer (Physica MCR 301). A transient heated needle (KD2 Pro, Decagon Devices, Inc., USA) was used to measure the thermal conductivity with $5 \%$ accuracy at a constant temperature. The thermal conductivity measurements are repeated ten times and the average values were reported. The electrical conductivity was measured using AB200 pH/Conductivity Meter (Fisher Scientific). The light transmission of all samples was measured with a Cary $50 \mathrm{UV}-\mathrm{V}$ is spectrophotometer, Agilent Technologies that is operating between 200 and $1100 \mathrm{~nm}$.

\section{Results and discussion}

\section{Characterization of nitrogen-doped graphene}

The FESEM image in Fig. 2 shows a uniform structure like a crumpled silk veil with porous and worm-like structures, while silk-like transparent NDG nanosheets are randomly stacked together. It could be observed that the two-dimensional graphene structures with high volume ratio and specific surface area are well retained after hydrothermal treatment with ammonia.

The specific surface area of the NDG sample was measured and the graph is shown in Fig. 3. The unique mesoporous structure of NDG contributes to the high specific surface area $\left(793 \mathrm{~m}^{2} / \mathrm{g}\right)$ which is higher than our prepared GO $\left(684 \mathrm{~m}^{2} / \mathrm{g}\right)$ with a uniform pore size distribution around $3-5 \mathrm{~nm}$. Over the synthesis of NDG sheets, besides the carbon atoms that were replaced by nitrogen atoms (most likely located on the reactive edge), ammonia can also react with graphene to form hydrogen cyanide and hydrogen $\left(\mathrm{C}+\mathrm{NH}_{3}=\mathrm{HCN}+\mathrm{H}_{2}\right)$.

The XRD patterns for the NDG and GO are shown in Fig. 4. Crystalline materials generally have welldefined XRD peaks, while amorphous materials result in a broad body ground with shallow peaks. The GO has a strong (001) peak at $2 \theta=9.71^{\circ}$ and $d$-spacing is 


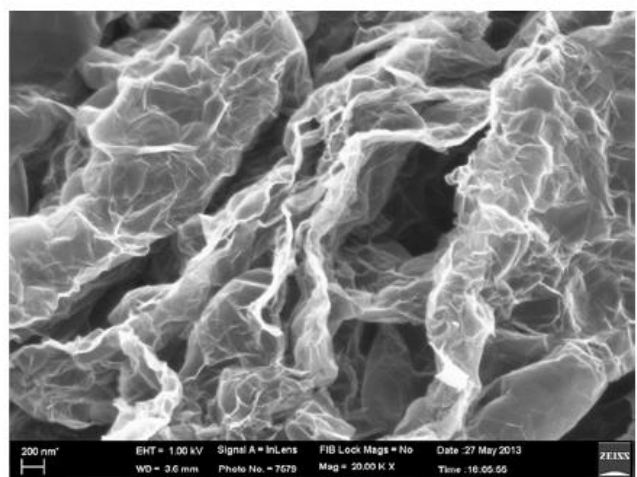

Fig. 2 FESEM image of NDG nanosheets

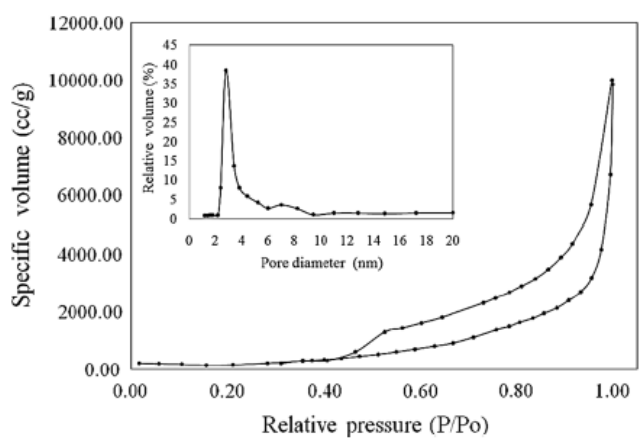

Fig. 3 Nitrogen adsorption/desorption isotherms of NDG. Inset is the $\mathrm{BJH}$ pore size distribution

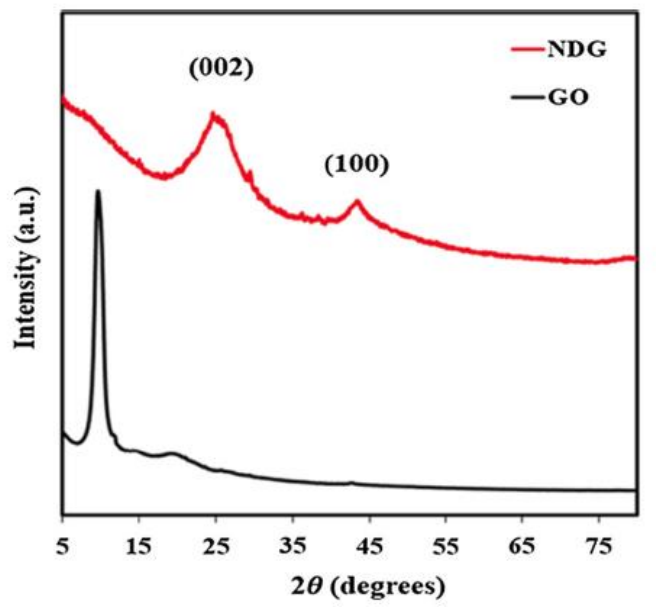

Fig. 4 XRD patterns of GO and NDG
$0.91 \mathrm{~nm}$, which is the preferred orientation of GO basal planes parallel to the sample plane (Blanton and Majumdar 2012; Liu et al. 2014).

In the XRD pattern for the NDG observed a peak at $2 \theta=24.5^{\circ}$ corresponding to the $(002)$ graphitic interlayer spacing, and another peak at $2 \theta=44^{\circ}$, corresponding to the (100) in-plane hexagonal atom arrangement. The NDG peaks are fairly small and broad, reflecting the defective nature of NDG, as well as the highly porous nature. It shows that only a few layers are stacked together, which result in a very low inter-layer spacing signal (Blanton and Majumdar 2012; Liu et al. 2014).

More descriptive details about its morphology were obtained from TEM. As can be observed in Fig. 5, NDG nanosheets were built with a regular crumpled surface with random stacking, which can be caused by the defective structure formed after exfoliation as well as the presence of foreign nitrogen atoms (Sheng et al. 2011). The very well-identified SAED spots and rings (inset of Fig. 5) showed a distinct hexagonal lattice, confirming the crystalline structure of NDG, but were slightly different from those of the specific graphene sheets due to doping and overlapping (Hernandez et al. 2008).

The single-sheet characteristics of the GO obtained were validated by AFM (Fig. 6). The thickness from the graphene sheet obtained was around $0.8 \mathrm{~nm}$, corresponding well with the reported apparent thickness of single-sheet graphene. Although there are sporadic multilayers in NDG, the AFM image demonstrates that NDG is uniform with an apparent thickness of about $1.8 \mathrm{~nm}$ (Fig. 6b), suggesting NDG is a fewlayer film.

FT-IR spectra were then used to analyze the chemical compositions of GO and NDG (Fig. 7). The main peaks in FT-IR spectrum of GO centered at $1046,1221,1412,1631,1728$, and $3400 \mathrm{~cm}^{-1}$ can be attributed to alkoxy $\mathrm{C}-\mathrm{O}$, epoxy $\mathrm{C}-\mathrm{O}$ and $\mathrm{C}-\mathrm{OH}$, carboxyl $\mathrm{O}=\mathrm{C}-\mathrm{O}$, aromatic $\mathrm{C}=\mathrm{C}, \mathrm{C}=\mathrm{O}$ (carboxylic acid and carbonyl moieties), and $\mathrm{O}-\mathrm{H}$ stretches, respectively, confirming the successful oxidation of graphite (Mehrali et al. 2013b). After solvothermal treatment, the content of these oxygen-containing groups in GO significantly decreased, while two new nitrogen-related peaks shown up at 1458 and $1550 \mathrm{~cm}^{-1}$ in the FT-IR spectrum of NDG, which can be preliminarily assigned to $\mathrm{C}-\mathrm{N}$ stretching and $\mathrm{N}-\mathrm{H}$ bending bonds of amide, respectively (Wu et al. 2012). 


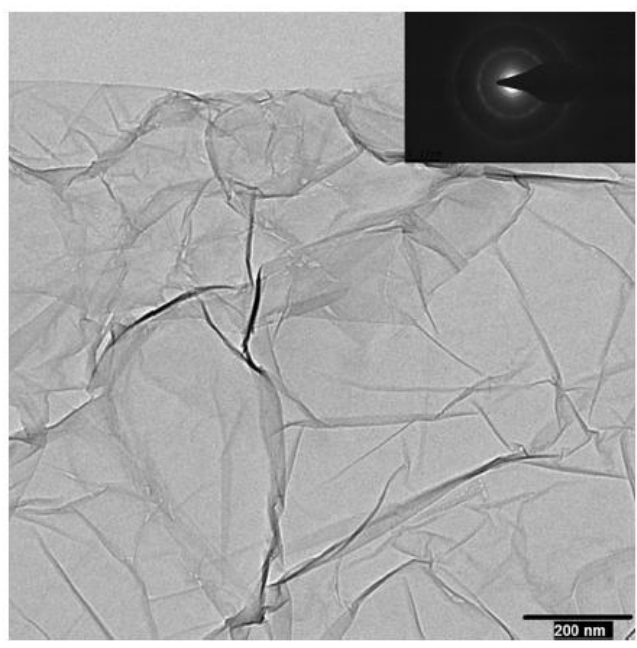

Fig. 5 TEM image of NDG and the corresponding SAED pattern (inset)

To help investigate the elemental composition and nitrogen bonding configurations in NDG, XPS measurements were performed. As shown in Fig. 8, there are three peaks centered at 284.2,399.3, and $532 \mathrm{eV}$, which are related to $\mathrm{C} 1 \mathrm{~s}, \mathrm{~N} 1 \mathrm{~s}$, and $\mathrm{O} 1 \mathrm{~s}$, respectively,

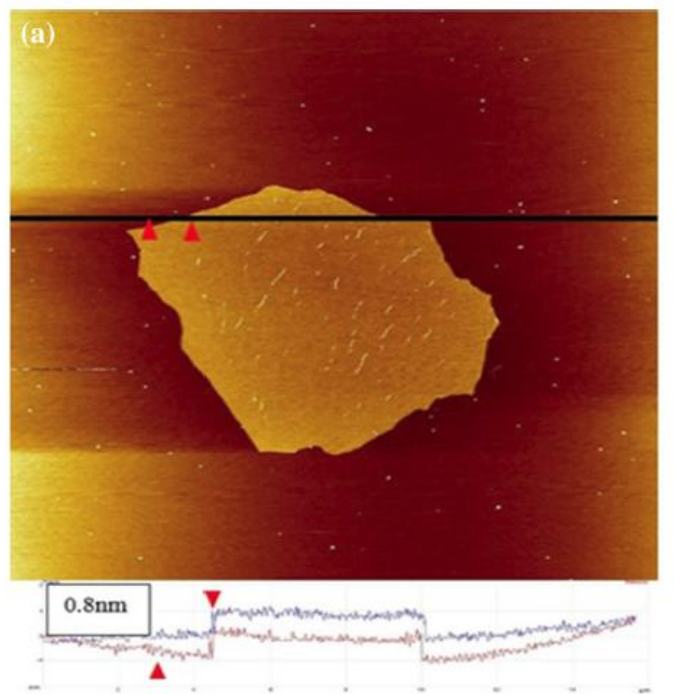

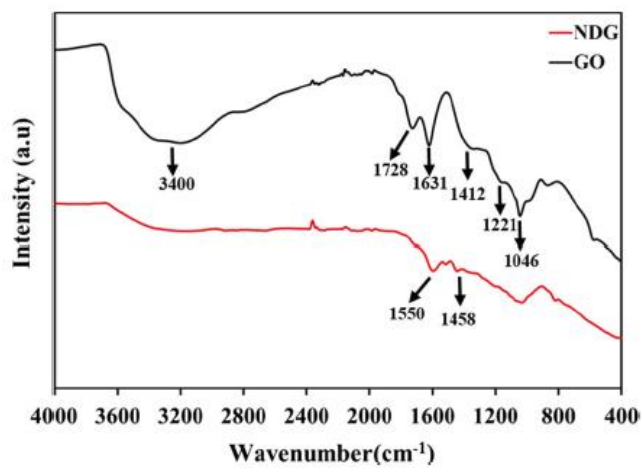

Fig. 7 FT-IR spectra of GO and NDG

and this shows that the incorporation of nitrogen within the graphene (Guo et al. 2013; Reddy et al. 2010)

The original GO has quite high oxygen content $(38.3 \%)$. Upon hydrothermal reduction, the carbon content raises up to $89.2 \%$, which is at the expense of the excellent reduction of oxygen content. This result suggests that the oxygen functionalities are actually eliminated mostly. It must be noted that the nitrogen content was zero within the initial GO, that was increased up to $2.64 \%$ in NDG structure.

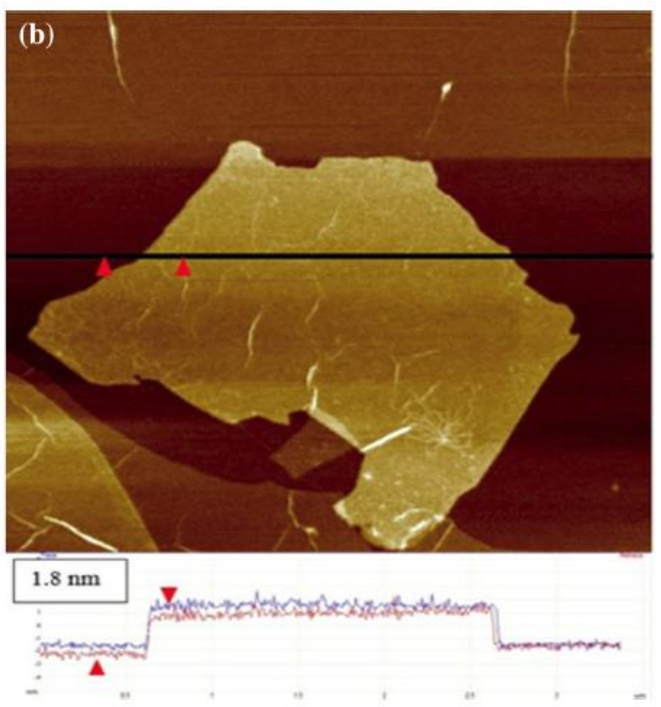

Fig. 6 AFM image of a GO and b NDG with corresponding height profile 
Figure 9 shows the C1s XPS spectra of GO and NDG. The full width at half maximum (FWHM) of NDG appeared to be narrower following reduction, suggesting an increased graphitic degree. A substantial level of oxidation was noticed in $\mathrm{GO}$, related to the carbon atoms in various functional groups: $\mathrm{C}-\mathrm{C}$ (284.7), $\mathrm{C}-\mathrm{O}$ (286.9), $\mathrm{C}=\mathrm{O}$ (288.1), and $\mathrm{COOH}$ (288.7 eV) (Mehrali et al. 2014d). Considerably, the peak intensities of oxygen-containing groups became considerably weaker in NDG, while it can be noticed that an additional peak showed up at $285.9 \mathrm{eV}$, which can be assigned to the $\mathrm{C}-\mathrm{N}$ bonds (Long et al. 2010).

The high-resolution N1 s XPS spectrum of NDG was then compiled in Fig. 10a. Generally, the peaks located at 398.2, 400.3, and $403.8 \mathrm{eV}$ are assigned to pyridinic-, pyrrolic-, and graphitic-forms of nitrogen atoms doped within the graphene structure, respectively (see Fig. 10b) (Mehrali et al. 2014c). It might be normally recognized that the covalent functionalization with amino groups may appear at the edge or defect sites of GO over the reduction process with ammonia; thus, the peak centered at $399.8 \mathrm{eV}$ could be assigned to amino nitrogen atom (Lin et al. 2010), which is in conjunction with the previous FT-IR result. FT-IR and XPS spectra can support that the pristine GO was properly reduced and successfully doped/modified with nitrogen atoms/amino group as a result of low-temperature solvothermal process, producing NDG successfully.

Raman spectroscopy is a powerful non-destructive technique to study carbonaceous materials including

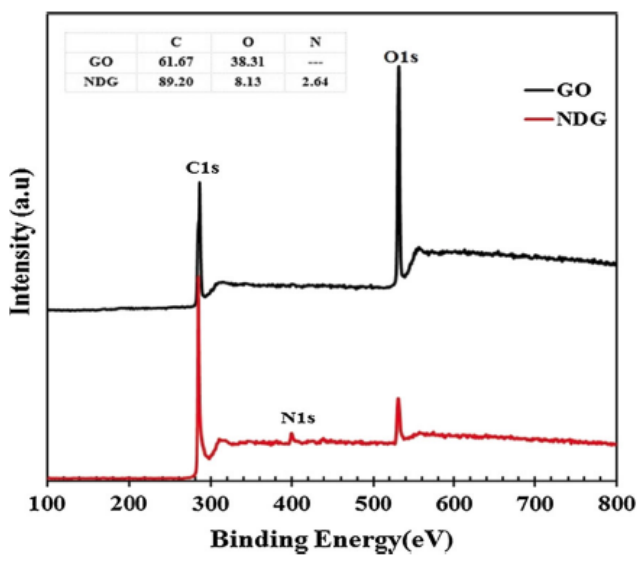

Fig. 8 Low-resolution XPS spectra of GO and NDG graphene. Raman characteristics of carbon materials are the $\mathrm{G}$ bands $\left(\sim 1580 \mathrm{~cm}^{-1}\right)$ and $\mathrm{D}$ bands $\left(\sim 1350 \mathrm{~cm}^{-1}\right.$ ) (Akhavan and Ghaderi 2012). The $\mathrm{G}$ bands are associate with the $E_{2 \mathrm{~g}}$ phonon of $\mathrm{C} \mathrm{sp}^{2}$ atoms and the $\mathrm{D}$ bands are breathing mode of $k$-point phonons of $A_{1 \mathrm{~g}}$ symmetry and are associated with local disorder and defects especially at the edges of graphene and graphite platelets (Akhavan 2010). Figure 11 shows the Raman spectra NDG and GO. The Raman spectrum for NDG gives two prominent peaks at 1354 and $1581 \mathrm{~cm}^{-1}$, which correspond to the $\mathrm{D}$ and $\mathrm{G}$ bands, respectively. Figure 11 shows the $\mathrm{G}$ line at about $1588 \mathrm{~cm}^{-1}$ and the D line at $1355 \mathrm{~cm}^{-1}$ for the GO. The intensity ratio between the $\mathrm{G}$ band and D band $\left(I_{\mathrm{D}} / I_{\mathrm{G}}\right)$ ratios for the samples GO and NDG were 0.77 and 0.94 , respectively. This shows that the nitrogen doping can make a lot of defects in the graphene structure, which can contribute to a highintensity D band (Vinayan and Ramaprabhu 2013). Moreover, the $2 \mathrm{D}$ band $\left(\sim 2700 \mathrm{~cm}^{-1}\right)$ can be used to distinguish graphene with different layers. A broader and up-shifted 2D peak, compared to the spectrum of single-layer graphene, indicates that the NDG is predominantly a few-layer graphene that was confirmed by AFM results.

The mass loss of GO was close to $5 \%$ at around $100{ }^{\circ} \mathrm{C}$, which is assigned to the elimination of water molecules captured within the GO structure (see Fig. 12). An instant mass loss of $20 \%$ taking place around $200{ }^{\circ} \mathrm{C}$ is attributed to the pyrolysis of the labile oxygen-containing groups in the forms of $\mathrm{CO}, \mathrm{CO}_{2}$, and steam. NDG reveals an extremely higher residue than GO. The main mass loss of NDG at $130-210{ }^{\circ} \mathrm{C}$ is because of the decomposition of residual oxygencontaining groups and $\mathrm{N}\left(\mathrm{CH}_{3}\right)_{2}^{+}$groups.

Stability evaluation of nitrogen-doped graphene nanofluid

The stability of the nanofluid was determined by measuring the sediment time by the UV-Vis spectrophotometer as is widely done in evaluating the relative concentration of the nanofluids. UV-Vis spectrophotometer method is based on Beer-Lambert's law, which states that the absorbance was directly proportional to the concentration of the nanosheet in the nanofluids.

Figure 13a shows the UV-Vis spectrophotograph of NDG colloidal suspension in DW. The maximum 

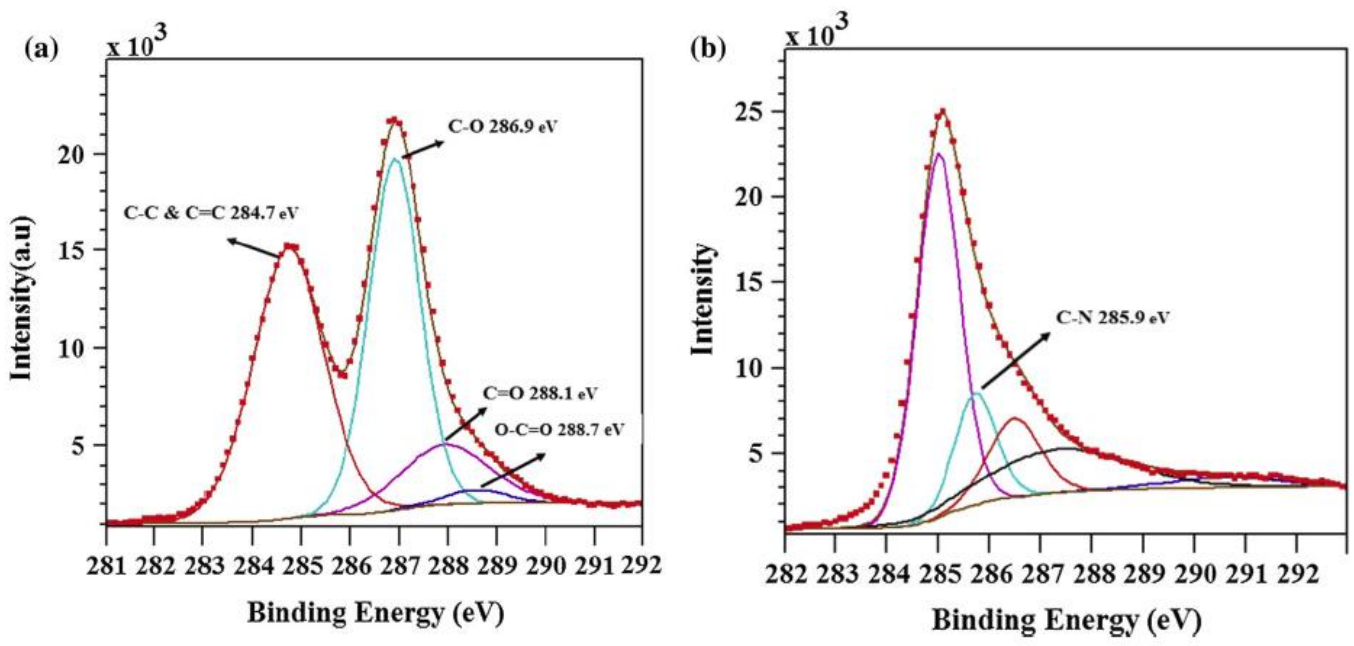

Fig. 9 The C1s XPS spectra of a GO and b NDG

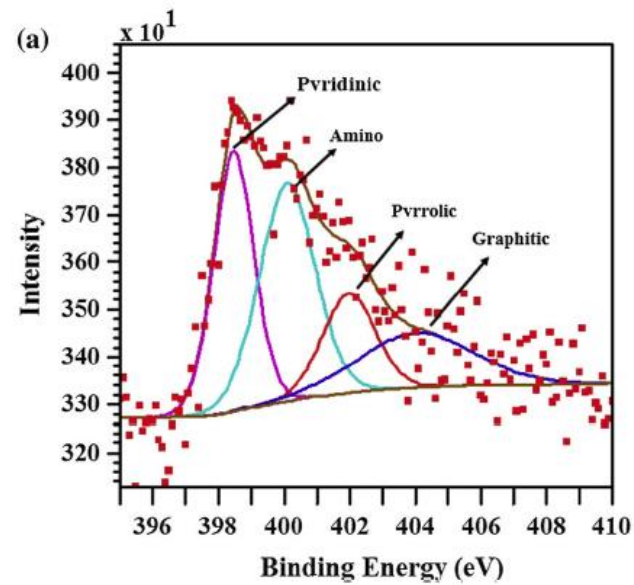

(b)

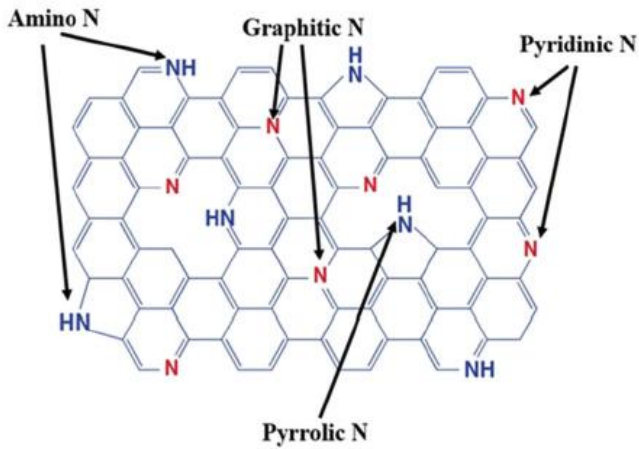

Fig. 10 a The N1s XPS spectrum of NDG and b schematic illustration of NDG. The various 'N' atoms represent the pyridinic N, pyrrolic $\mathrm{N}$, graphitic $\mathrm{N}$, and amino group in graphene structure

linear absorption wavelength is located at $275 \mathrm{~nm}$, which is the same for all concentrations. It can satisfy Beer's law and indicates that NDG was dispersed well in the base fluid. It shows that the suspension can be considered as a stable nanofluid. It also shows that the characteristic bands corresponding to additional absorption are due to the 1D van Hove singularities (Aravind et al. 2011). To measure the relative concentration of the suspensions with the sediment time, the same sample of DW and NDG nanofluids was used as a reference to eliminate the absorbance of nanofluid.

The dispersed nanosheet in the base fluids was influenced by gravity, as well as by particle-particle and particle-fluids interactions (Mehrali et al. 2014b). Therefore, the concentration of the nanofluids changes from the initial state of preparation and it is important to study the stability of the nanofluids. Figure $13 \mathrm{~b}$ 


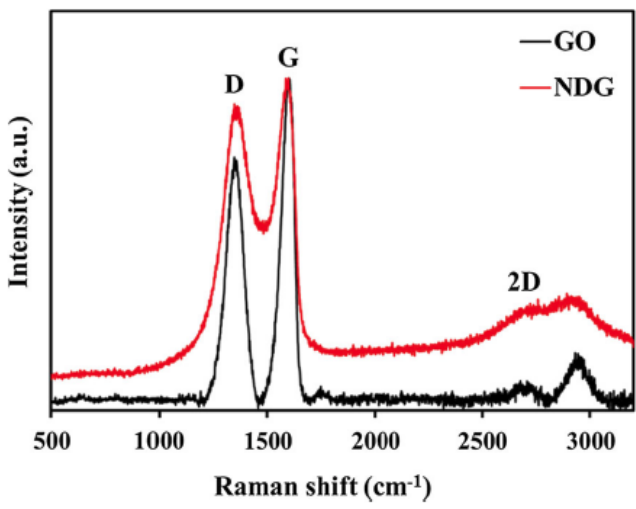

Fig. 11 Raman spectra of GO and NDG

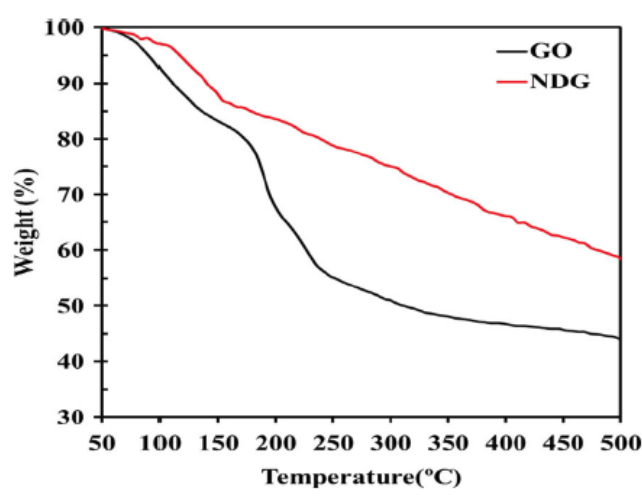

Fig. 12 TGA curves of GO and NDG

shows the colloidal stability of the NDG nanofluids during 200 days. The concentration of NDG nanofluids was decreased, which was attributed to sedimentation and particle agglomeration after long periods. Figure 13b shows that the relative concentration of nanofluid at $0.01 \mathrm{wt} \%$ was almost steady for 200 days.

It shows that the colloidal stability of the NDG nanofluids for the concentrations of 0.02, 0.04, and $0.06 \mathrm{wt} \%$ remains relatively constant and was only reduced by 10,16 , and $20 \%$, respectively.

The colloidal stability of NDG nanofluids could be predicted from the zeta potential values of the nanosheet dispersed in DW, which closely related to its electrophoretic properties of nanofluids. A welldispersed suspension can be obtained with a zeta potential of more than $+30 \mathrm{mV}$ or less than $-30 \mathrm{mV}$ (high surface charge density) to generate strong repulsive forces (Mehrali et al. 2014b). The zeta potential value at $\mathrm{pH} 8$ is $-46.3 \mathrm{mV}$, which is in line with the excellent stability found by UV-Vis studies (Mehrali et al. 2014b). Additionally, the particle size distribution of nanosheet is another important factor and it is suggested that average particle size is not sufficient to characterize a nanofluid due to the nonlinear relations involved between particle size and thermal transport. It is also known that particle shape is an effective parameter on the electrical conductivity. The particle size distribution on NDG nanofluids was at $412.4 \mathrm{~nm}$ for $60 \mathrm{~min}$ ultrasonication time.

Thermal properties analysis

The dependence of thermal conductivity on temperature is presented in Fig. 14, which shows that the effective thermal conductivity increases with increasing temperature and NDG concentration. The enhancement of thermal conductivity for NDG nanofluids is between 22 and $37 \%$. The principal mechanism of thermal conductivity enhancement can be explained by the stochastic motion of the nanosheets. Based on the literature, there is an interfacial resistance between the nanosheets and base fluid that affects the thermal conductivity of the nanofluids. The suspended nanosheets in the base fluid experience stochastic bombardment from the ambient liquid molecules through the raising of temperature. This causes irregular motion, called Brownian motion (Xuan et al. 2006). Brownian motion is related to nanosheet concentration and fluid temperature (Hemmat Esfe et al. 2014). This irregular motion of the nanosheets is induced from micro-mixing or microconvection inside the base fluid. For these reasons, the energy exchange between the base fluid and the nanosheets is enhanced and the thermal conductivity is enhanced (Xuan et al. 2006).

Rheological behavior analysis

The rheological behaviors of NDG nanofluids are presented in Fig. 15. The viscosity versus shear rate is measured in the range of $20-60{ }^{\circ} \mathrm{C}$ and tested over the shear rate range of $0.1-500 \mathrm{~s}^{-1}$. It can be seen that the viscosity reduces between 51.2 and $51.5 \%$ as temperature rises. Thermal movement of molecules, brownian motion intensifies, and intra-molecular 
(a)

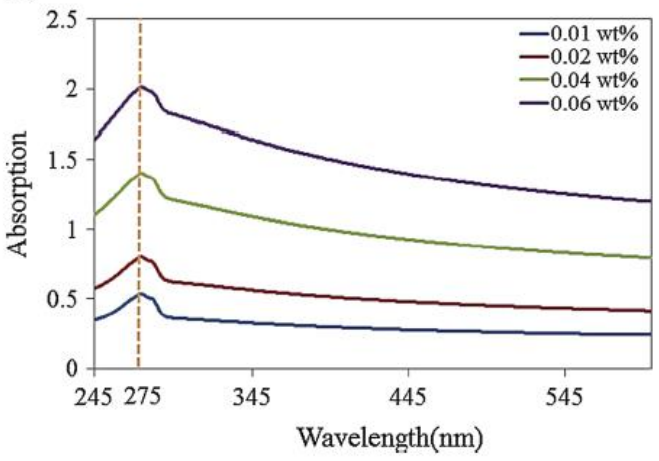

(b)

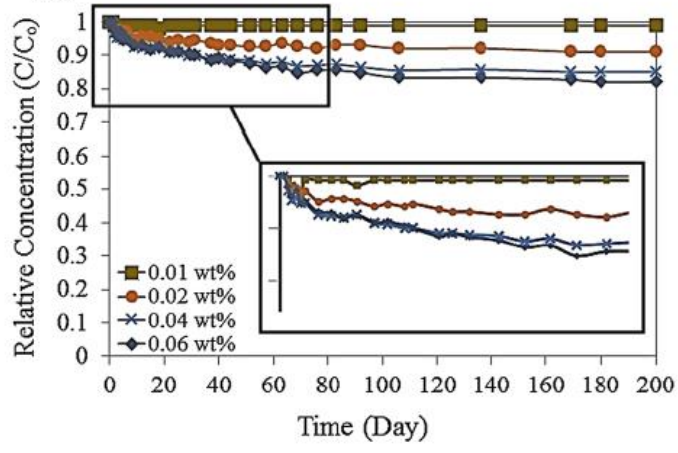

Fig. 13 a UV-Vis spectrophotometer of nanofluids at different concentrations and wavelengths and b relative supernatant particle concentration of nanofluids with sediment time

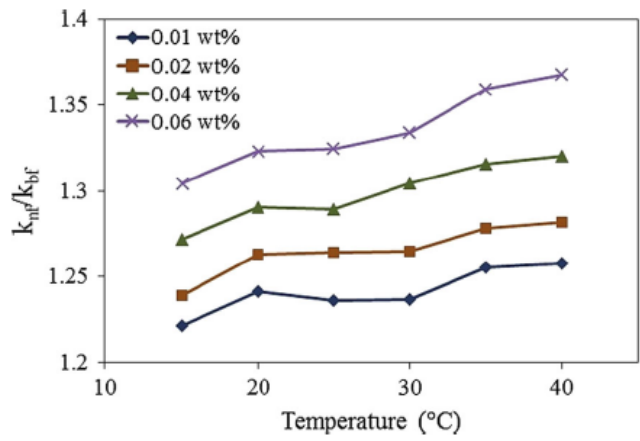

Fig. 14 Effective thermal conductivity of NDG nanofluids as a function of temperature for several concentrations

interactions become weakened with the rise of temperature. Additionally, it is shown that the variation in concentration increases the viscosity; however, other investigated parameters such as temperature and concentration have an important influence on viscosity behavior and heat transfer properties of nanofluids.

Electrical conductivity of aqueous nitrogen-doped graphene nanofluid

Figure 16 shows the electrical conductivity of the NDG nanofluids with respect to weight percentage for different temperatures. As shown Fig. 16, the electrical conductivity increases with increasing the NDG nanosheet concentration. The maximum enhancement of electrical conductivity is $308.16,667.34,1311.56$, and $1814.96 \%$ at the loading of $0.01,0.02,0.04$, and
$0.06 \mathrm{wt} \%$, respectively. The electrical conductivity also increases almost linearly with temperature, especially at higher concentration. However, the results show that electrical conductivity depends on temperature much less than on NDG concentration.

The increment in electrical conductivity of the NDG nanofluids compare to the base fluid is a consequence of the net charge effect on the NDG nanosheets and the relevant EDL interactions around each particle which is the major importance of colloidal stability of suspensions. As shown in Fig. 17, EDL can exist around each particle and include two parts: (a) an inner region (Stern layer) where the ions are strongly bound and (b) an outer (diffuse) region where they are less firmly associated (Minea and Luciu 2012).

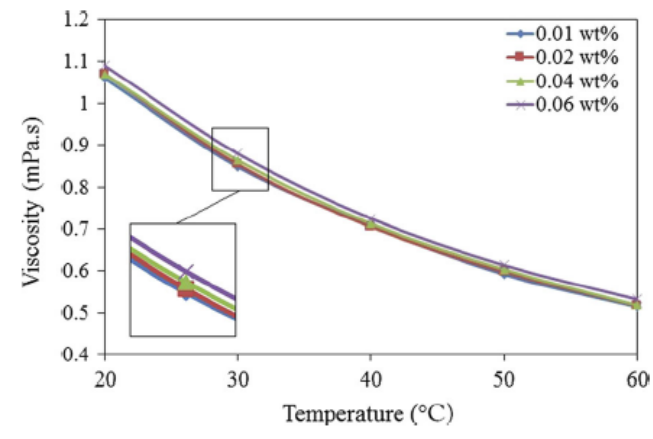

Fig. 15 Viscosity as a function of temperature for several concentrations of NDG nanofluids

\section{Link to Full-Text Articles :}

